\title{
Significance of Understanding Function of Glymphatic System to Manage Practical Clinical Problems of the Elderly
}

\author{
Andrzej Brodziak $^{1 *}$, Agnieszka Wolińska ${ }^{2}$, Alicja Różyk - Myrta ${ }^{2}$ \\ ${ }^{l}$ Institute of Occupational Medicine and Environmental Health, Sosnowiec, Poland \\ ${ }^{2}$ Institute of Medical Sciences, University of Applied Sciences, Nysa, Poland
}

*Corresponding Author: Andrzej Brodziak, Institute of Occupational Medicine and Environmental Health, Sosnowiec, Poland, Email: andrzejbrodziak@wp.pl

\begin{abstract}
The authors set themselves the task of explaining the significance of understanding the function of the glymphatic system for the clinical management of practical problems of the elderly. Since the glymphatic clearance system of the brain was discovered not long ago and investigations have only recently begun, the authors first present essential data about the structural elements and organization of the system. They devote considerable attention to the phenomenon of increased activity of the system during sleep. They also mention the conceptual model of the neurovascular unit, integrating the anatomic and metabolic interactions between neurons, vascular components, and glial cells. Next they refer to the significance of decreased clearance performed by this system in the development of neurogenerative diseases and pathology caused by traumatic brain injury. The authors then present the impairment of glymphatic function in the aging brain. They also consider the contemporary possibilities of objective estimation of the function of the glymphatic system. These preliminary sections finally enable discussion of the contemporary possibilities to take into account the knowledge about the glymphatic system in clinical practice.
\end{abstract}

Keywords: glymphatic system, neurogenerative diseases, traumatic brain injury, aging brain, sleep, geriatrics

\section{BACKGROUND}

The discovery some years ago of the unknown system clearing the brain from waste metabolites produced during its functioning was an astonishing event. Nadia Aalling Jessen in her frequently cited review paper wrote: "The glymphatic system is a recently discovered macroscopic waste clearance system that utilizes a unique system of perivascular tunnels, formed by astroglial cells, to promote efficient elimination of soluble proteins and metabolites from the central nervous system" [1].

It occurred that this system works mainly when we are sleeping. Moreover, only a few years ago it occurred that simultaneous cognitive performance and clearing waste metabolites from brain tissue not only in humans but also in most animal species is impossible.

It can be summarized in an intuitive way that brain tissue cells must be shrunk during sleep to significantly increase the volume of the space containing waste metabolites. Information processing and clearing must take place in alternating periods of time. These findings contribute to understanding the physiological role of sleep.

The glymphatic system is contemporarily one of the most studied functional systems of the human body [1-6]. Much work has also already been devoted to the significance of impaired function of the glymphatic system in the development of dementia and other neurodegenerative diseases [7, 8, 9]. Some researchers have emphasized that the function of the glymphatic system is impaired in the elderly $[10,11,12]$.

The data gathered recently are relevant for clinicians. Knowledge of the function of the glymphatic system can be used to improve geriatric care. In this paper we try to present these implications. In order to do so, it is necessary to first present the elements of the anatomical structure and activities of this system.

\section{Structural Elements AND ORGANIZATION OF THE GLYMPHATIC SYSTEM}

Only an astute student of human anatomy realizes how important the function of the 
lymphatic system is in all organs of the human body. Unnecessarily, often toxic waste metabolic by-products are drained from the body's cells into the interstitial fluid (ISF) and from there to numerous tiny lymphatic vessels. Collective lymphatic vessels drain these materials into the venous system. However, there are no lymphatic vessels in the brain and spinal cord. Cleaning metabolites from nervous tissue is done through a complex system consisting of several pathways $[1,2]$.

It is necessary to remember that in the brain, apart from vasculature and two other fluid compartments - intracellular and interstitial, there is yet the cerebrospinal compartment and fluid (CSF). CSF is produced in the choroid

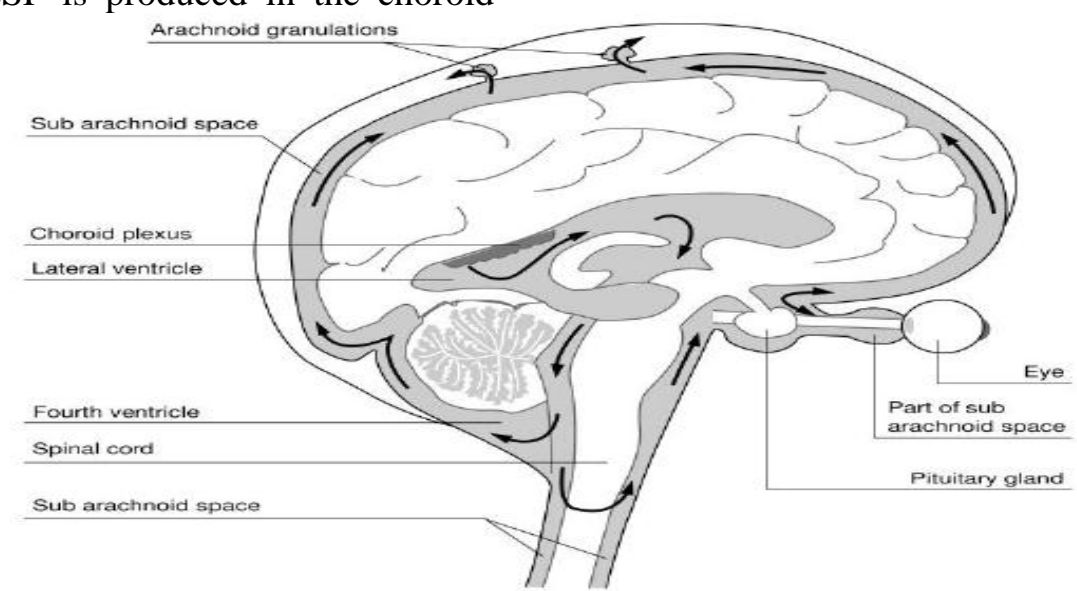

Figure1. Scheme of the cerebrospinal fluid circulation

Because the accumulation of biochemical components in the cerebrospinal space should be strictly regulated, blood is separated from brain tissue by the blood-brain and bloodcerebrospinal barriers [1]. These gateways function on the basis of specific endothelial cells [1].

Another specific feature of brain vessels is related to the astrocytic end feet. They surround the arterioles and venules of the brain plexus of the brain and flows through its basal cisterns. Therefore, unlike other organs, the brain and spinal cord are somehow immersed in the cerebrospinal fluid that surrounds it and as it turns out, it acts as a flushing, cleansing agent $[1,2,4,6$,$] .$

During sleep, subarachnoid cerebrospinal fluid rapidly recirculates through the cisternal compartments and penetrates the brain along the paravascular spaces surrounding cerebral arteries and permeates to the interstitial fluid. In turn, ISF flows along the paravascular spaces surrounding large caliber cerebral veins. We recall the structure of the cerebrospinal space by means of Figure 1.

\section{- $[1,2,4,6]$. We try to il Figure 2.}

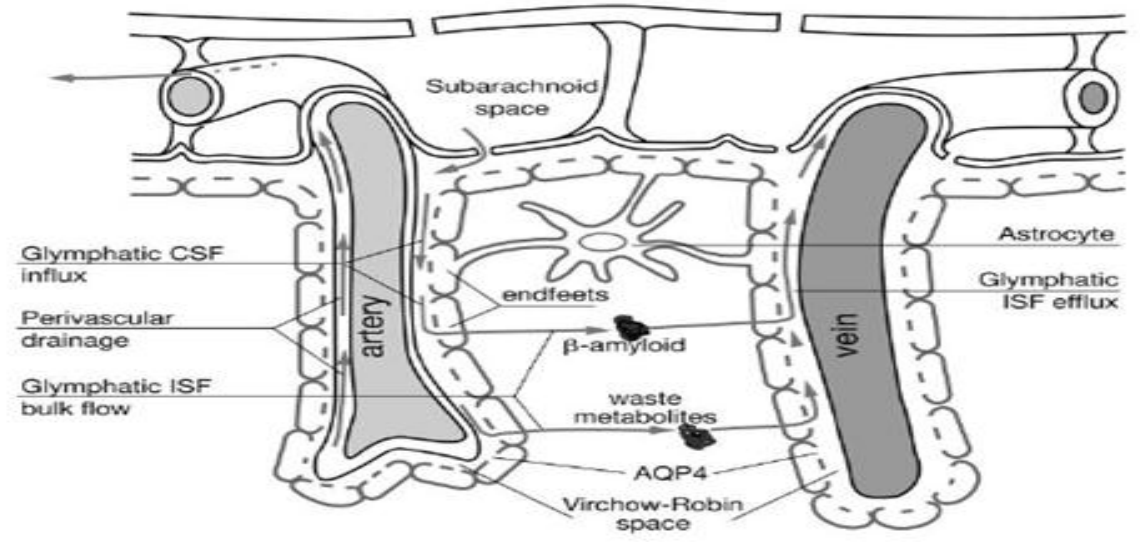

Figure2. Intuitive presentation of the pathways of the glymphatic system. 
CSF passes through the para-arterial space that surrounds the arteries to the vascular end feet of the astrocytes. It is possible by reason of the existence of so-called "water channels" called aquaporin 4 (AQP4) in the membrane of vascular endfeet of astrocytes $[2,4]$. In this way, the metabolic by-products flow from CSF through the astrocytes to the interstitial space surrounding the veins. Finally, ISF exits the nervous tissue through the paravenous route and reaches the lymphatic vessels in the neck transferring the metabolic by-products to systemic circulation.

The glymphatic system is active mainly when we are sleeping and its action is suspended in wakefulness. Xie et al. demonstrated that the paravenous interstitial clearance route changes during sleep-wake cycles [3]. They noticed that "natural sleep or anesthesia are associated with a $60 \%$ increase in the interstitial space, resulting in a striking increase in convective exchange of cerebrospinal fluid with interstitial fluid" [3]. These authors are convinced that" the restorative function of sleep may be a consequence of the enhanced removal of potentially neurotoxic waste products that accumulate in the awake central nervous system" [3]. Ferdinand K. Hui remarks that "the pump mechanism of volume expansion and subsequent contraction may be an important factor in brain homeostasis and a teleological reason for sleep" [4].

Jessen et al. note that norepinephrine is responsible for suppressing the glymphatic system during wakefulness [1]. Lundgaard et al. recently found that brain lactate concentration is higher during wakefulness than in sleep and that brain lactate is an excellent biomarker of the sleep-wake cycle. Brain lactate is inversely correlated with glymphatic-lymphatic clearance [13]. Glucose that is not fully oxidized can be exported as lactate via the glymphatic-lymphatic fluid transport system [13].

The brain, due to its considerable need for essential substances and energy, requires effective control of cerebral blood flow (CBF). It is ensured by tight coupling between neuronal activity, CBF, and metabolism [14]. A regional $\mathrm{CBF}$ increase in response to neuronal activation is observed using neuroimaging techniques such as functional magnetic resonance imaging. The mediators are nitric oxide, lactate and ion channels and astrocytes are involved as well. The conceptual model integrating the anatomic and metabolic interactions between neurons, vascular components, and glial cells is called the neurovascular unit [14].

\section{The Significance OF Clearance PERFORMED BY THE GLYMPHATIC SYSTEM AND ITS RELATION TO NEUROGENERATIVE DISEASES}

Proper functioning of the nervous system requires effective cleaning of metabolic byproducts. Ineffective elimination of toxic substances is one of the causes of neurodegenerative diseases [7, 8]. Former investigations were focused on the intracellular degradation of such substances. Recent studies have demonstrated that toxic proteins are present in interstitial and cerebrospinal fluids [1]. In Alzheimer's disease amyloid deposits are found in perivascular spaces and the perivascular space is enlarged. The drainage of beta-amyloid through perivascular pathways is impaired in this clinical state [7]. Decreased activity of the glymphatic system seems to be an essential risk factor of this neurodegenerative disease. Susceptibility to neurodegenerative diseases is also associated with sleep disorders [16].

Mendelsohn et al state that "clinical studies suggest that the quality and duration of sleep may be predictive of the onset of $\mathrm{AD}$, and that quality sleep may significantly reduce the risk of AD" [15]. Deformation of the perivascular space is also found in vascular forms of dementia [1].

\section{Pathological Changes of the GLYMPHATIC SYSTEM IN TRAUMATIC BRAIN INJURY}

It is known that traumatic brain injury is a predisposition to dementia and Alzheimer's disease [1]. Even moderate but repeated head injuries lead to neurogenerative disease [1]. There are several biomarkers of traumatic brain injury like C-tau peptide [17]. The betaamyloid peptide accumulates in greater amounts following traumatic brain injury [17]. The levels of these biomarkers correlate with the severity of the injury [17]. Traumatic brain injury causes glial scars. In animal models of repetitive brain injury CSF circulation and the clearance of cerebrospinal fluid was decreased [1]. The reduction in glymphatic function persisted 28 days after the induced injury [1]. In addition, traumatic brain injury is characterized by the dislocation of AQP4 channels [1]. They are 
shifted from the vascular end feet of astrocytes to the parenchymal parts of the cells. This dislocation is similar to the changes found in the aging brain [10]. Impairment of the function of the glymphatic system is also noted after embolic ischemic stroke, however, this damage is transient [18]. Thus, different brain injuries like stroke, subarachnoid bleeding and repetitive traumatic events impair the function of the glymphatic system [1].

\section{IMPAIRMENT OF THE GLYMPHATIC FUNCTION IN THE AGING BRAIN}

Investigations of the function of the glymphatic system in young and old mice models demonstrated that it is decreased by $80-90 \%$ in aged individuals [10]. The aging brain is characterized by the dislocation of AQP4 channels [10]. The loss of polarity of AQP4 is noted. These channels, instead of being located within the end feet, appear in the parenchymal parts of astrocytes. Hence, in the elderly the main pathways of water flow from cerebrospinal fluid to interstitial fluid is impaired. Furthermore, a $66 \%$ decrease in cerebrospinal fluid formation and a decrease in its pressure of $27 \%$ was noted $[11,12]$. One of the driving forces of glymphatic flow is the pulsatility of cerebral arterioles. In the aging brain the brain vessel walls become stiffer, reducing the interstitial flux [10]. Impairment of glymphatic function in the elderly causes the accumulation of amyloid deposits in perivascular spaces, which also reduces the passage of CSF to ISF. Alzheimer's disease, Parkinson's disease, Huntington's disease, front temporal dementia and traumatic encephalopathy are examples of neurodegenerative diseases that arise in old age, co-occurring with impaired function of the glymphatic system in the elderly [1]. Cognitive dysfunction in the elderly occurs as a result of all these pathological transformations.

\section{CONTEMPORARY POSSIBILITIES OF OBJECTIVE ESTIMATION OF THE FUNCTION OF THE GLYMPHATIC SYSTEM}

The action of the system was first demonstrated by very sophisticated techniques. The neuroscientists used so-called two-photon microscopy, which enabled them to assess the periarterial flow of markers injected into the cerebrospinal fluid space [1].

It was found that "in vivo 2-photon imaging of glymphatic function showed that the cerebrospinal fluid influx in the awake state was reduced by $90 \%$ compared to anesthetized mice" [1]. It was demonstrated then that during sleep the volume fraction of interstitial space expands by about $80 \%$ [1]. The administration of a contrast agent injected into the cerebrospinal space enables real-time tracking of the flow through all the pathways of the glymphatic system [1].

Nadia Aalling Jessen quoted Yang et al. and remarks that "intrathecal lumbar injections, which are routinely used in clinical myelographic studies, provide a viable route to assess the basic parameters of glymphatic function" [1,19]. Studies using contrast-enhanced MRI were developed next and provided useful data to evaluate the glymphatic pathways $[18,20]$. Contemporary clinical neuroscientists are trying to develop a safe and minimally invasive technique based on MRI imaging [21].

\section{Elements OF KNOWLEDGE ABOUT THE GLYMPHATIC SYSTEM THAT MAY AFFECT Current Clinical Practice}

Since it was discovered that the efficacy of the glymphatic system declines significantly in old age and that impairment of its function increases the probability of neurodegenerative diseases, it is worth considering whether current knowledge is indicative for activities in clinical practice.

It is possible to indicate which activities would be desirable. It would certainly be useful to develop methods enabling objective assessment of the performance of the glymphatic system and consequently to determine which easily observable clinical signs indicate with a high probability existing impairment of the system.

Among the factors improving the function of the glymphatic system would be the possibility to increase the production of CSF and its pressure. It would also be desirable to accelerate the exchange between the CSF and ISF. It would be beneficial to increase the ISF outflow to systemic lymphatic vessels and the circulatory system. There is also the concept of the treatment targeted to eliminate the amyloid- $\beta$ deposits or prevent its accumulation. Sun BL et al. discuss such possibilities [22].

Investigations related to the possibility of modifying the flow of water and dissolved waste substances through aquaporin-4 channels and astrocyte cell bodies have just begun $[23,24]$.

It seems that in the future the results of these investigations will also have practical implications.

It would be beneficial to have drugs that increase the elasticity and pulsation of cerebral arterioles. This pulsation is considered as the pump (type I) of the glymphatic system. Sun BL et al. sugest that some vasoactive drugs can have 
positive impact on the glymphatic clearance [22]. These authors discuss the results of animal experiments on the effects of such drugs as Rolipram, Sildenafil, Ciloztasol [22].

Because during sleep the volume of ISF increases significantly and afterwards decreases in the awakened state (pump - type II), the question is whether it is beneficial to wake up older people several times. At present, this is only a hypothetical possibility derived from the cited above opinion of Ferdinand K. Hui [4].

Lee $H$. et al. found that "waste, including $A \beta$, removal was most efficient in the lateral position during sleep (compared with the prone position), which mimics the natural resting/sleeping position of rodents" [25].

Probably in the future the effect of continuous theta burst stimulation (cTBS) will be useful in clinical practice. cTBS is a pattern of repetitive transcranial magnetic stimulation (rTMS) which has been shown to enhance sleep efficacy and reduce sleep-associated emotional and cognitive dysfunction [26]. The effect of cTBS on the glymphatic system was recently assessed on a mouse model of sleep deprivation by Liu DX et. al. [26]. These investigators found that mice diprivated of sleep had reduced influx through the perivascular space, disturbed AQP4 polarization and demonstrated anxiety-like behaviors. cTBS restored the loss of AQP4 polarization and improved anxiety-like behavior in those mice. The authors are convinced that cTBS has the potential to protect against neuronal dysfunction induced by sleep disorders.

Wostyn et al. considering the structure of the glymphatic system inferred that observation of the function of this system in patients with glaucoma would probably provide support for their hypothesis that CSF circulatory dysfunction may contribute to the pathogenesis of glaucomatous damage [27]. Just like Alzheimer's disease, glaucoma might be caused by an imbalance between the production and clearance of neurotoxins, including amyloid- $\beta$ [24]. Schain et al. argue that impairment of the glymphatic system can be involved in the pathogenesis of migraine [28]. Hitscherich et al. consider the possibilities of so-called "osteopathic manipulative treatment" to improve the function of the glymphatic system. They note that these manipulations could concentrate on opening myofascial transition areas, maximalization of diaphragmatic movement and augmenting lymphaticovenous outflow [28].
These authors emphasize the significance of body posture and respiration patterns. They remark that glymphatic transport activity is most efficient in the right lateral position compared with the supine or prone positions. Body position is known to influence sympathetic tone, with sympathetic tone being lower in the right lateral position compared with that in the left lateral position. Body position is also known to affect respiratory function, particularly during the night sleep cycle. The flow of CSF is increased during inspiration, particularly when performed during forced breathing. They maintain that "it is conceivable, then, that aspiratory thoracic pressure may also contribute to glymphatic transport activity." [28]

\section{Conclusions}

- Since investigations of the recently discovered system which performs the cleaning of metabolism by-products from brain tissue started not long ago, it is necessary to disseminate the knowledge about the structure and function of the glymphatic system as well as data about its importance in relation to the development of neurogenerative diseases and pathology caused by traumatic brain injury.

- Knowledge of the inevitable impairment of the function of the glymphatic system in old age is important for geriatric care.

- Despite the very recent beginning of investigations of the function of the glymphatic system, many of the gathered data are already important for day-to-day clinical practice.

\section{REFERENCES}

[1] Jessen NA, Munk AS, Lundgaard I, Nedergaard M. The Glymphatic System: Beginner's Guide. Neurochem Res 2015; 40(12):2583-99.

[2] Nedergaard M. Garbage truck of the brain. Science. 2013; 340 (6140): 1529-1530

[3] Xie L, Kang H, Xu Q, Chen MJ, Liao Y et al. Sleep drives metabolite clearance from the adult brain. Science, 2013; 342(6156): 10.1126 /science.1241224.

[4] Hui FK. Clearing your mind: A glymphatic system? World Neurosurgery. 2015; 83(5):715 717

[5] Ratner V, Gao Y, Lee H, Elkin R, Nedergaard M, Benveniste $\mathrm{H}$, Tannenbaum A. Cerebrospinal and interstitial fluid transport via the glymphatic pathway modeled by optimal mass transport. Neuroimage 2017; 152:530-537. 
[6] Benveniste H, Lee H, Volkow ND. The glymphatic pathway. Neuroscientist 2017; Jan 1:107385841769 1030. doi: 10.1177/10738584 17691030. [Epub ahead of print]

[7] Tarasoff-Conway JM, Carare RO, Osorio RS, et. al. Clearance systems in the brainimplications for Alzheimer disease. Nat Rev Neurol 2016;12(4): 248

[8] Bakker EN, Bacskai BJ, Arbel-Ornath M, et al. Lymphatic clearance of the brain: perivascular, paravascular and significance for neurodegener ative diseases. Cell Mol Neurobiol 2016; 36(2):181-94.

[9] Peng W, Achariyar TM, Li B, et al. Suppressi on of glymphatic fluid transport in a mouse model of Alzheimer's disease. Neurobiol Dis 2016; 93:215-25.

[10] Kress BT, Iliff JJ, Xia M, et al. Impairment of paravascular clearance pathways in the aging brain. Ann Neurol. 2014; 76(6):845-61.

[11] Chen RL, Kassem NA, Redzic ZB, et al. Agerelated changes in choroid plexus and bloodcerebrospinal fluid barrier function in the sheep. Exp Gerontol 2009; 44(4):289296.

[12] Fleischman D, Berdahl JP, Zaydlarova J, et al. Cerebrospinal fluid pressure decreases with older age. PLoS One. 2012; 7: e52664.

[13] Lundgaard I, Lu ML, Yang E, et al. Glymphatic clearance controls state-dependent changes in brain lactate concentration. J Cereb Blood Flow Metab 2017; 37(6):2112-2124.

[14] Venkat P, Chopp M, Chen J. New insights into coupling and uncoupling of cerebral blood flow and metabolism in the brain. Croat Med J 2016;57(3):223-8.

[15] Mendelsohn AR, Larrick JW. Sleep facilitates clearance of metabolites from the brain: glymphatic function in aging and neurodegenerative diseases. Rejuvenation Res 2013; 16(6):518-23.

[16] Eugene AR, Masiak J. The neuroprotective aspects of sleep. MEDtube Sci. 2015; 3(1):35-40.

[17] Plog BA, Dashnaw ML, Hitomi E, et al. Biomarkers of traumatic injury are transported from brain to blood via the glymphatic system. J Neurosci 2015; 35(2):518-26.

[18] Gaberel T, Gakuba C, Goulay R, et al. Impaired glymphatic perfusion after strokes revealed by contrast-enhanced MRI: a new target for fibrinolysis? Stroke. 2014; 45(10):3092-6.

[19] Yang L, Kress BT, Weber HJ, et al. Evaluating glymphatic pathway function utilizing clinically relevant intrathecal infusion of CSF tracer. J Transl Med 2013; 11:107.

[20] Iliff JJ, Lee H, Yu M, et al. Brain-wide pathway for waste clearance captured by contrast-enhanced MRI. J Clin Invest. 2013; 123(3):12 99-309.

[21] Taoka T, Masutani Y, Kawai H, et al. Evaluation of glymphatic system activity with the diffusion MR technique: diffusion tensor image analysis along the perivascular space (DTI-ALPS) in Alzheimer's disease cases. Jpn J Radiol. 2017; 35(4):172-178.

[22] Sun BL, Wang LH, Yang T et al. Lymphatic drainage system of the brain: A novel targetfor intervention of neurological diseases. Prog Neurobiol. 2017; pii: S0301-0082(17)30062-X. doi: 10.1016/j.pneurobio.2 017.08.007. [Epub ahead of print]

[23] Leitão RA, Sereno J, Castelhano JM, et al. Aquaporin- 4 as a new target against methampheta mine-induced brain alterations: focus on the neurogliovascular unit and motivational behavior. Mol Neurobiol. 2017; doi: 10.1007/s120 35- 0170439-0. [Epub ahead of print]

[24] Lan YL, Chen JJ, Hu G, Xu J, Xiao M, Li S. Aquaporin 4 in astrocytes is a target for therapy in Alzheimer's disease. Curr Pharm Des. 2017 Jul 14. doi: 10.2174/138161282366617071414 4844. [Epub ahead of print]

[25] Lee H, Xie L., Yu M, et al. The effect of body posture on brain glymphatic transport. $\mathbf{J}$ Neurosci. 2015; 35(31):11034-11044.

[26] Liu DX, He X, Wu D, et al. Continuous theta burst stimulation facilitates the clearance efficiency of the glymphatic pathway in a mouse model of sleep deprivation. Neurosci Lett. 2017; 653:189-194.

[27] Wostyn P, Van Dam D, Audenaert K, Killer HE, De Deyn PP, De Groot V. A new glaucoma hypothesis: a role of glymphatic system dysfunction. Fluids Barriers CNS. 2015; 12: 16.

[28] Schain AJ, Melo-Carrillo A, Strassman AM, Bu rstein R. Cortical spreading depression closes paravascular space and impairs glymphatic flow: Implications for migraine hea dache. Neurosci 2017; 37(11):2904-2915.

[29] Hitscherich K, Smith K, Cuoco JA, Ruvolo KE, Mancini JD, Leheste JR, Torres G. The glymphatic-lymphatic continuum: Opportunities for osteopathic manipulative medicine. J Am Osteopath Assoc. 2016; 116(3):170-7.

Citation: Andrzej Brodziak, Agnieszka Wolińska, Alicja Różyk - Myrta. Significance of Understanding Function of Glymphatic System to Manage Practical Clinical Problems of the Elderly. ARC Journal of Neuroscience. 2018; 3(2):15-20. doi:dx.doi.org/10.20431/2456-057X.0302005.

Copyright: (c) 2018 Authors. This is an open-access article distributed under the terms of the Creative Commons Attribution License, which permits unrestricted use, distribution, and reproduction in any medium, provided the original author and source are credited. 